\title{
5-Hydroxyeicosatetraenoic Acid
}

National Cancer Institute

\section{Source}

National Cancer Institute. 5-Hydroxyeicosatetraenoic Acid. NCI Thesaurus. Code C558.

A lipoxyg enase metabolite of arachidonic acid and an intermediate in the pathway of leukotriene synthesis. 5-hydroxyeicosatetraenoic acid (5-HETE) is also a modulator of tubuloglomerular feedback, one of the mechanism the kidney uses to regulate glomerular filtration rate. Along with leukotriene B4, 5-HETE is often used as biomarker in evaluating eicosanoid metabolism and disease state. 